\title{
Heat And Mass Transfer On Mhd Oscillatory Slip Flow In A Channel Filled With Porous Medium
}

\author{
Disu Akeem B., Ishola Christie Y. \& Olorunnishola Toyin \\ School of Science and Technology, National Open University of Nigeria \\ Victoria Island, Nigeria
}

\begin{abstract}
This study was conducted to investigate heat and mass transfer of unsteady MHD oscillatory slip flow of optically thin fluid through a channel filled with saturated porous medium and non-uniform walls temperature. The governing equations of oscillatory fluid flow were non-dimensionalised, simplified and solved. The closed-form solutions were obtained for the velocity, temperature and concentration. The numerical computations were presented graphically to show the salient features of the fluid flow, heat and mass transfer characteristics. The skin friction, Nusselt number and Sherwood number were also analyzed.
\end{abstract}

Key words: Chemical reaction, Porous medium, MHD oscillatory flow, Navier slip

\section{Introduction}

The study of oscillatory fluid flow in a porous channel has been receiving considerable attention in the last recent years due to its applications in soil mechanics, ground water hydrology, irrigation, drainage, water purification processes, absorption and filltration processes in chemical engineering. Also, the Navier slip flow regime has been receiving attention of many researchers, because of its applications in modern science, technology and industrialization [1]. Owing to these applications, Makinde and Osalusi [2] presented the effects of slip conditions on the hydromagnetic steady flow in a channel with permeable boundaries. The effects of Navier slip condition on the lower wall of unsteady MHD of a viscous fluid in a planner channel with filled with saturated porous was reported by Mehmood and Ali [3]. It was assumed that the Navier slip boundary condition effect depends on the shear stress of both lower and upper walls of a channel by Eegunjobi and Makinde [1]. Recently, Adesanya and Makinde [4] investigated the navier slip condition on the upper and lowers of pulsatile flow of oscillatory fluid through a channel filled with porous media.

The study of combined heat and mass transfer with chemical reaction on oscillatory fluid flow is of great practical applications in design of chemical processing equipments, formation and dispersion of flog, food processing and cooling of tower. The effects of chemical reaction on MHD oscillatory flow past through a porous plate with viscous dissipation and heat sink was presented by Vijaya and Viswannadh [5]. Rabi, Gouranger and Artpita [6] investigated the effects of chemical reaction on MHD oscillatory flow through a porous medium bounded by vertical porous plate with heat source and sore effect.

In view of these studues, we present chemical reaction effects on MHD oscillatory slip flow in an optically thin fluid through a channel filled with saturated porous medium and non-uniform walls temperature.

\section{Mathematical Formulation}

Consider the unsteady laminar slip flow of an electrically conducting, heat generating, optically thin and chemical reacting oscillatory fluid in a channel filled with saturated porous medium under the influence of an externally applied homogenous magnetic field and radiative heat transfer.

$$
\begin{aligned}
& \frac{\partial u}{\partial t^{*}}=-\frac{1}{\rho} \frac{\partial P}{\partial X}+v \frac{\partial^{2} P}{\partial Y^{2}}+\frac{g \beta_{T}\left(T-T_{0}\right)}{\rho}+\frac{g \beta_{C}\left(C-C_{0}\right)}{\rho}-\frac{H_{0}^{2} U}{\rho}-\frac{v}{K^{*}} U \\
& \frac{\partial T}{\partial t^{*}}=\frac{k}{\rho c_{P}} \frac{\partial^{2} T}{\partial Y^{2}}-\frac{1}{\rho c_{P}} \frac{\partial q}{\partial Y} \\
& \frac{\partial C}{\partial t^{*}}=D \frac{\partial^{2} C}{\partial Y^{2}}-K_{R}\left(C-C_{0}\right)
\end{aligned}
$$

The boundary conditions relevant to the problem are taken as follows: 


$$
\begin{aligned}
& U=\gamma^{*} \frac{\partial u}{\partial y}, T=T_{0}, C=C_{0} a t Y=0 \\
& U=\eta^{*} \frac{\partial u}{\partial y}, T=T_{1}, C=C_{1} a t Y=d
\end{aligned}
$$

where $U$ is the axial velocity, $t$ the time, $T$ the fluid temperature, $C$ fluid concentration, $P$ the pressure, $\mathrm{g}$ the gravitational force, $\mathrm{q}$ the radiative heat flux, $\beta_{T}$ the coefficient of volume expansion due to temperature, $\beta_{C}$ the coefficient of volume expansion due to concentration, $C_{P}$ the specific heat at constant pressure, $D$ thermal diffusivity, $\mathrm{k}$ the thermal conductivity, $v$ the kinematic viscosity, $H_{0}^{2}$ the uniform magnetic field, $K^{*}$ the porous medium permeability coefficient and $\rho$ the density of the fluid.It is assumed that both walls temperature $T_{0}, T, \mathrm{~T}$ are high enough to induce radiative heat transfer. It is assumed that the fluid is optically thin with a relatively low

density and the radiative heat flux is given by cogley et al [7] as follow:

$$
\frac{\partial q}{\partial y}=\chi^{2}\left(T_{0}-T_{1}\right)
$$

where $\chi$ is the mean radiation absorption coefficient. The following dimensionless variable and parameters are introduced

$$
\begin{aligned}
& (x, y)=\frac{1}{d}(X, Y), u=\frac{u}{U_{0}}, t=\frac{U_{0} t^{*}}{d}, P=\frac{P^{*} d}{v U_{0}}, \theta=\frac{T-T_{0}}{T_{1}-T_{0}}, \phi=\frac{C-C_{0}}{C_{1}-C_{0}}, S c=\frac{D}{U_{0} d} \\
& \operatorname{Re}=\frac{U_{0} d}{v}, G r=\frac{g \beta_{T}\left(T-T_{0}\right) d^{2}}{v U_{0}}, G c=\frac{g \beta_{C}\left(C-C_{0}\right) d^{2}}{v U_{0}}, D a=\frac{K^{*}}{d^{2}}, s=\frac{1}{D a}, K_{r}=\frac{K_{R}}{U_{0}} \\
& \gamma=\frac{\gamma^{*}}{d}, \eta=\frac{\eta^{*}}{d}, H=\frac{H_{0}^{2} d^{2}}{\rho v}, P e=\frac{\rho c_{P} U_{0} d}{k}, N=\frac{4 \chi d^{2}}{k}
\end{aligned}
$$

where $U_{0}$ is the flow mean velocity, $G r$ the Grasshof number, $G c$ the solutal Grasshof number, $\mathrm{H}$ the Hartmann number, $\mathrm{N}$ is the radiation parameter, Pe the Peclet number, Re the Reynold number Da the Darcy number, $s=\frac{1}{D a}$ porous medium shape factor parameter, and $\lambda$ is the dimensionless frequency of oscillation The equations (1) to (5) in dimensionless form reduce to

$$
\begin{aligned}
& \operatorname{Re} \frac{\partial u}{\partial t}=-\frac{\partial P}{\partial x}+\frac{\partial^{2} u}{\partial y^{2}}-(s+H) u+G r \theta+G c \phi \\
& P e \frac{\partial \theta}{\partial t}=\frac{\partial^{2} u}{\partial y^{2}}+N \theta \\
& \frac{\partial \phi}{\partial t}=S c+\frac{\partial^{2} \phi}{\partial y^{2}}-K_{r} \phi
\end{aligned}
$$

The boundary condition becomes

$$
u=\gamma \frac{\partial u}{\partial y}, \theta=0, \phi=0 \quad \text { on } y=0
$$


$u=\eta \frac{\partial u}{\partial y}, \theta=1, \phi=1 \quad$ on $y=1$

\section{Method Of Solution}

In order to solve equations (7) - (9) with respest to the boundary conditions (10) for purely oscillatory flow $-\frac{\partial P}{\partial x}=\lambda e^{i \omega t}, u(y, t)=u_{0}(y) e^{i \omega t}, \theta(y, t)=\theta_{0}(y) e^{i \omega t}, \phi(y, t)=\phi_{0}(y) e^{i \omega t}$

where $\lambda$ is a constant and $\omega$ is the frequency of oscillation.

Substituting equation (11) into (7) - (10), we obtain:

$$
\begin{aligned}
& \frac{d^{2} u_{0}}{\partial y^{2}}-m_{1}^{2} u_{0}=-\left(\lambda+G r \theta_{0}+G c \phi_{0}\right) \\
& \frac{d^{2} \theta_{0}}{\partial y^{2}}+m_{2}^{2} \theta_{0}=0 \\
& \frac{d^{2} \phi_{0}}{\partial y^{2}}-m_{3}^{2} \phi_{0}=0
\end{aligned}
$$

together with boundary conditions

$$
\begin{array}{ll}
u_{0}=\gamma \frac{\partial u_{0}}{\partial y}, \theta_{0}=0, \phi_{0}=0 & \text { on } y=0 \\
u_{0}=\eta \frac{\partial u_{0}}{\partial y}, \theta_{0}=1, \phi_{0}=1 & \text { on } y=1
\end{array}
$$

where $m_{1}=\sqrt{s+H+i \omega t}, m_{2}=\sqrt{N-i \omega P e}$ and $m_{3}=\sqrt{\frac{1}{S c}\left(K_{r}+i \omega t\right)}$

Equations (12) -(15) are solved and the solution for fluid velocity, temperature and concentration are given as follows:

$$
\begin{aligned}
& \theta(y, t)=\frac{\sin \left(m_{2} y\right)}{\sin \left(m_{2}\right)} e^{i \omega t} \\
& \phi(y, t)=\frac{\sinh \left(m_{2} y\right)}{\sinh \left(m_{2}\right)} e^{i \omega t} \\
& u(y, t)=-\frac{\left(-1+\cosh \left(m_{1}\right)+\sinh \left(m_{2}\right)\right) A_{2}}{4 A_{2}\left\{2 \sinh \left(m_{1}\right)+\left(\cosh \left(m_{1}\right)+\sinh \left(m_{1}\right)\right) m_{1}-m_{1}\left(\cosh \left(m_{1}\right)-\sinh \left(m_{1}\right)\right)\right\}}
\end{aligned}
$$

The shear stress, the coefficient of the rate of heat transfer and the rate of mass at point in the fluid can be expressed by

$$
\tau=\mu \frac{\partial u}{\partial y} \quad, N u=-\frac{\partial \theta}{\partial y} \quad, S h=-\frac{\partial \phi}{\partial y}
$$

The skin friction $(\tau)$, the Nusselt number $(N u)$ and the Sherwood number $(S h)$ are expressed as follow:

$$
\begin{aligned}
& \tau=\frac{1}{4} \frac{A_{1}\left(\operatorname { c o s h } \left(m_{1}(y-1)+\sinh \left(m_{1}(y-1) m_{1}-\sinh \left(m_{1} y\right) m_{1}-\cosh \left(m_{1} y\right)\right)\right.\right.}{\sinh \left(m_{1}\right) A_{1}\left(m_{1}^{2}-1\right)} \\
& N u=-\frac{m_{2} \cos \left(m_{2} y\right)}{\sin \left(m_{2}\right)}, S h=\frac{m_{3} \cosh \left(m_{3} y\right)}{\sinh \left(m_{3}\right)}
\end{aligned}
$$




\section{Discussion Of Results}

We obtained the exact solutions of equations (12) - (14) subject to boundary condition (14). As an accurate check, setting Gc $=0$ and neglecting equation (14), the work of Adesanya and Makinde [7] is fully recovered. Therefore, study in [7] is a special form of the present paper. Numerical computation was performed on the exact solutions and the results were illustrated graphically in Fig 1-16 to show the interesting features of significant parameter on velocity, temperature and concentration distribution. Through the computations we employed default values $\mathrm{t}=0, \lambda=1, \omega=1, \mathrm{H}=2, \mathrm{~s}=1, \mathrm{k}=2, \mathrm{Pe}=0.7, \mathrm{Gr}=1, \mathrm{Gc}=1, \mathrm{Sc}=1$ and $\sigma=1=$ 1 unless otherwise stated on the graph.

Figure 1 illustrates that the presence of transverse magnetic field produces a resistive force on the fluid flow. This force is called Lorentz force, which slows down the motion of the fluid. It is observed that the increases in the frequencies of oscillation decrease the velocity as shown in Figure 2.

Increases in thermal and solutal Grashof numbers significantly increase the boundary layer thickness which resulted into rapid enhancement of fluid velocity as presented in Figures 3 and 4. Figure 5 depicts the variation of fluid velocity with inverse of the Darcy parameter, the graph shows that an increase in inverse of the Darcy parameter decreases the fluid velocity.

Figure 6 depicts that the increases in the radiation parameter increases the fluid velocity. Figures 7, 8 and 9 illustrate the variation of chemical reaction parameter $(\mathrm{K})$, Reynolds number $(\mathrm{Re})$ and Pelect number respectively. An increase in any of the parameter produces a reduction on the fluid velocity.

Figure 10 shows that the velocity profile variation with cold wall slip parameter.The graph shows that fluid velocity increases with an increase in the cold wall slip parameter $(\gamma)$ which enhancing the fluid flow mean that the slip parameter has the tendency to reduce friction forces which increases the fluid velocity. It is observed in Figure (11) that increase in the heated wall slip parameter $(\sigma)$ decreases the fluid velocity.

Figure 12 illustrates increase in the Schmidt number increasing the fluid velocity slightly. The temperature profile is plotted in Figure 13 and 14, it can be observed that the fuid temperature increases transversely with maximum value at the lower wall and maximum value at the upper wall. However, a general increase in the fuid temperature is observed with an increase in any of radiation or Peclet number.

Figure 15 illustrates the decrease in concentration for increasing chemical reaction parameter which indicates that the diffusion rate which can be changed tremendously by chemical reaction. Figure 16 depicts variation of concentration profle and Schmidt number. This shows that increase in Schmidt number increases the mass transfer.

Tables1\& 2 Show the effects of cold and heated wall on the skin friction. These show that an increase in any of the heat wall increases the shear stress and the transfer within the channel. Table 3 shows the effect of Pelect number on the Nusselt number. It is observed that an increase in Pelect number enhancing the rate of heat transfer on the wall.

Tables 4 and 5 show that effect of Schmit number and chemical reaction parameter on Sherwood number .It can been seen that an increase in eithet Schmit number or chemical reaction parameter increases the Sherwood number.

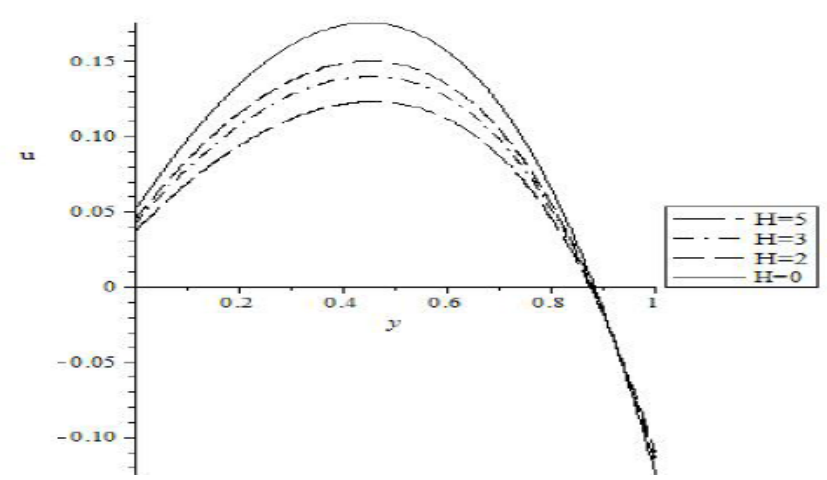

Figure 1: Velocity profile for different values of $\mathrm{H}$ 


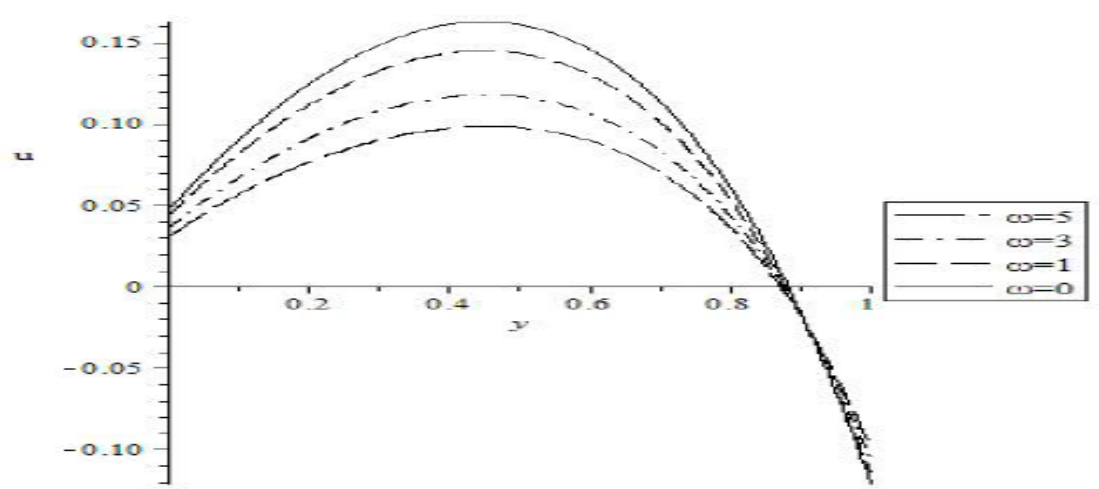

Figure 2: Velocity profile for different values of $\omega$

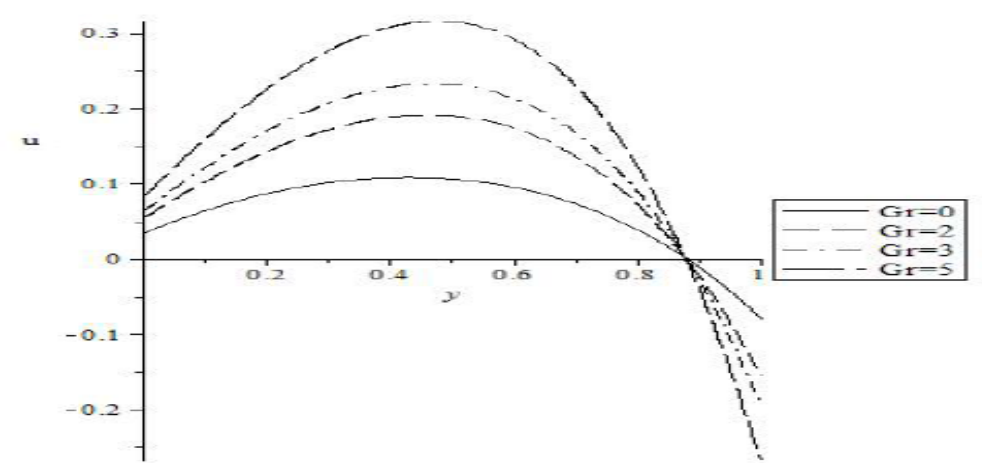

Figure 3: Velocity profile for different values of $G r$

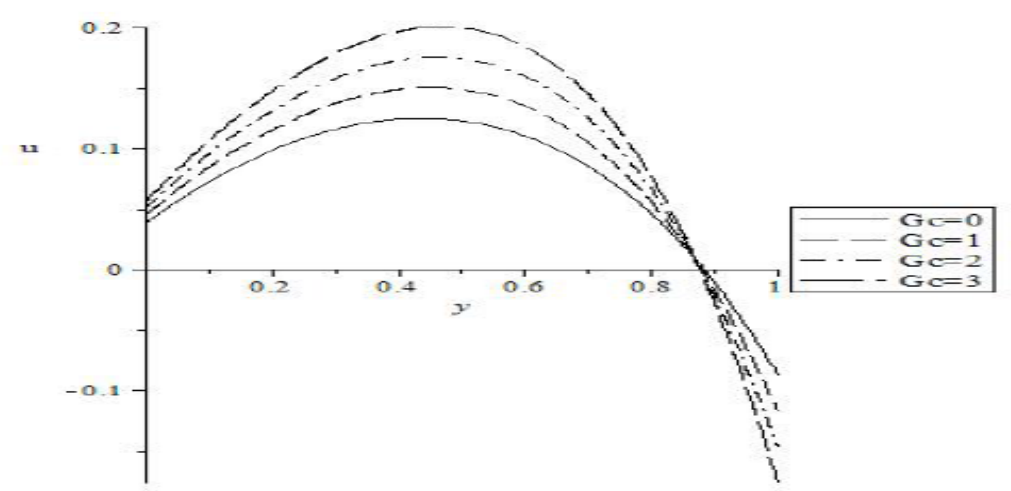

Figure 4: Velocity profile for different values of $G c$

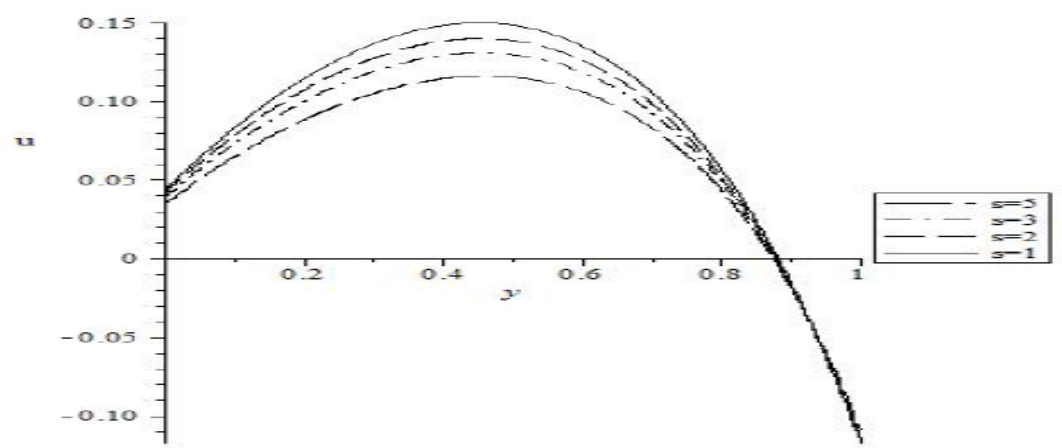

Figure 5: Velocity profile for different values of $s$ 


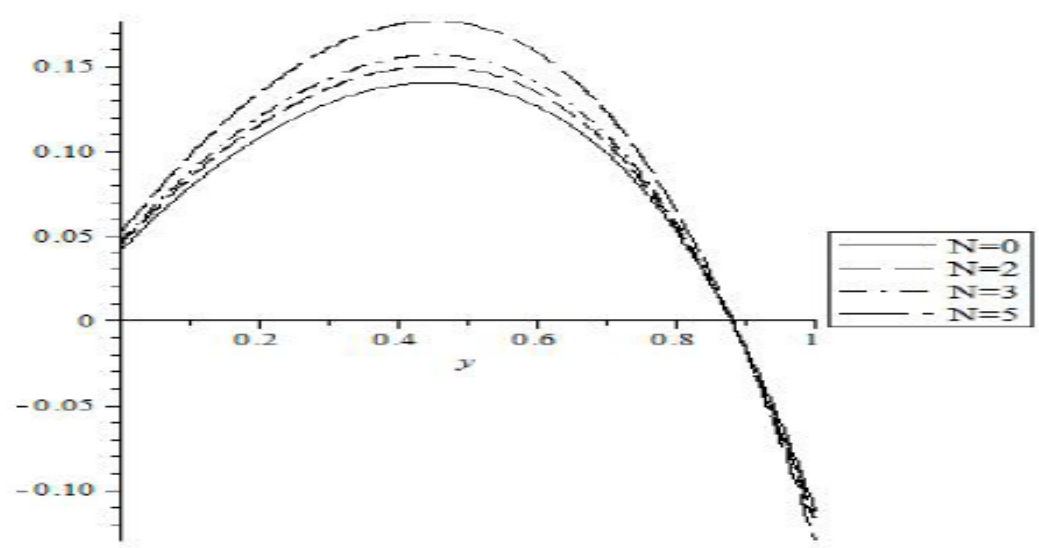

Figure 6: Velocity profile for different values of $N$

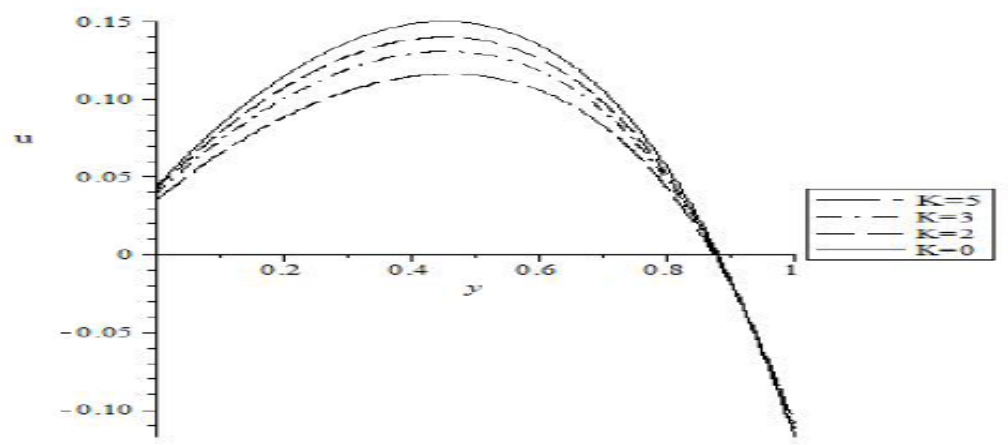

Figure 7: Velocity profile for different values of $K$

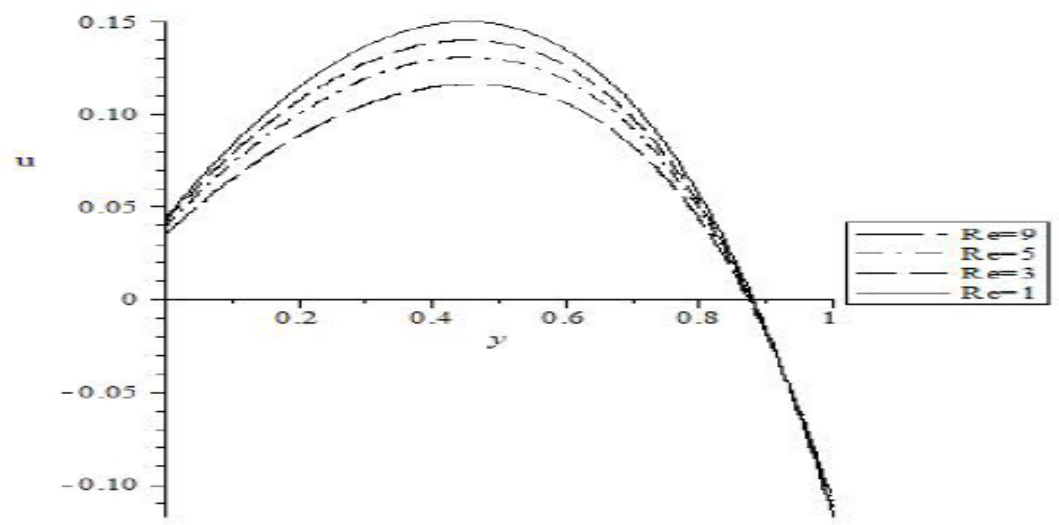

Figure 8: Velocity profile for different values of Re

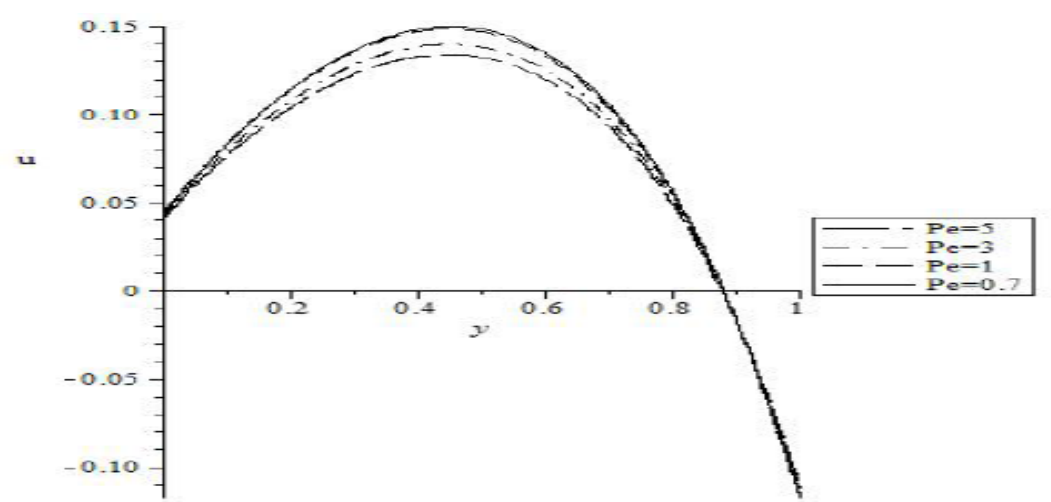

Figure 9: Velocity profile for different values of $P e$ 


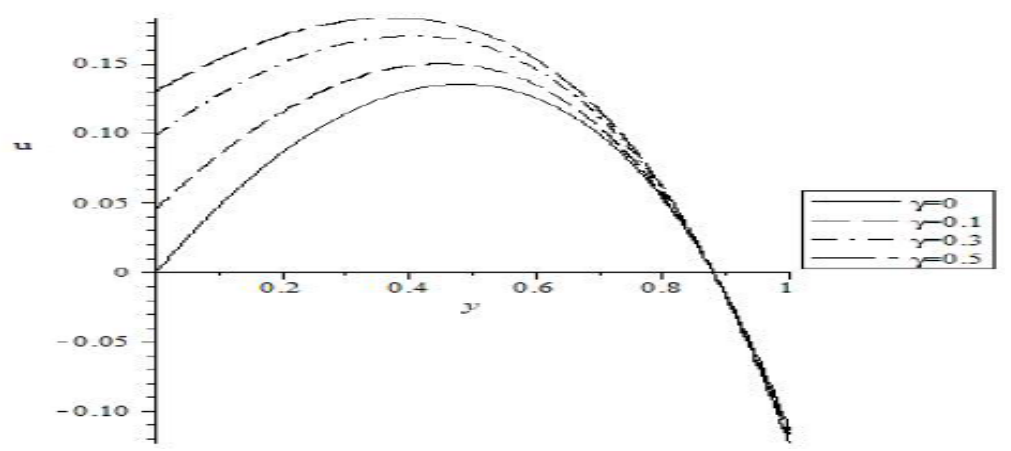

Figure 10: Velocity profile for different values of $\gamma$

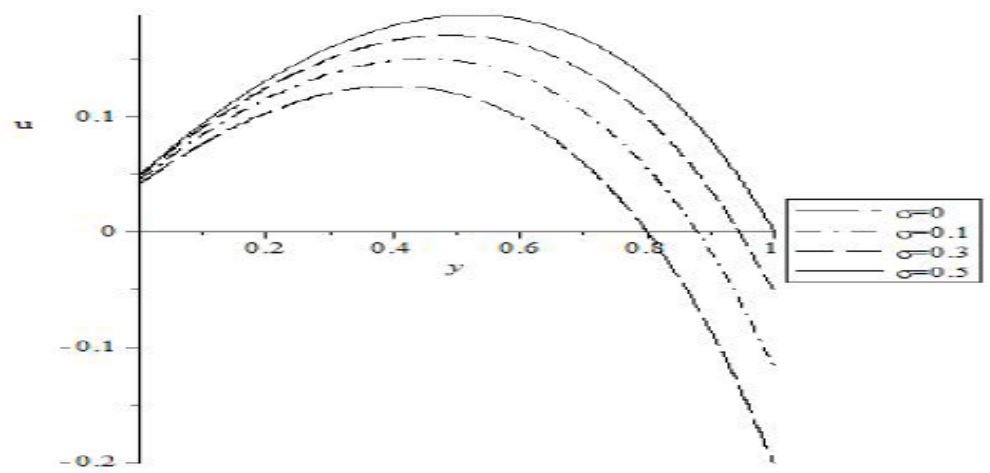

Figure 11: Velocity profile for different values of $\eta$

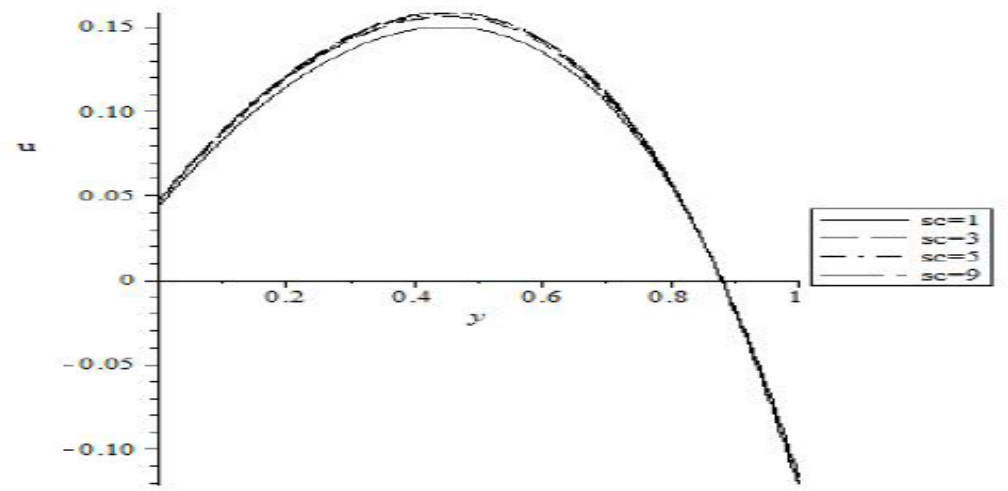

Figure 12: Velocity profile for different values of $S c$

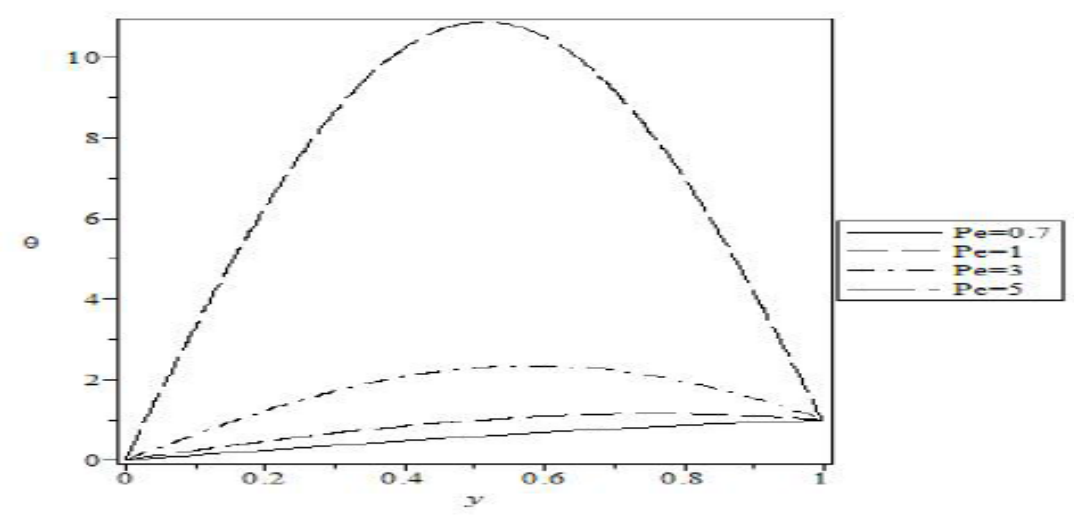

Figure 13: Temperature profile for different values of $P e$ 


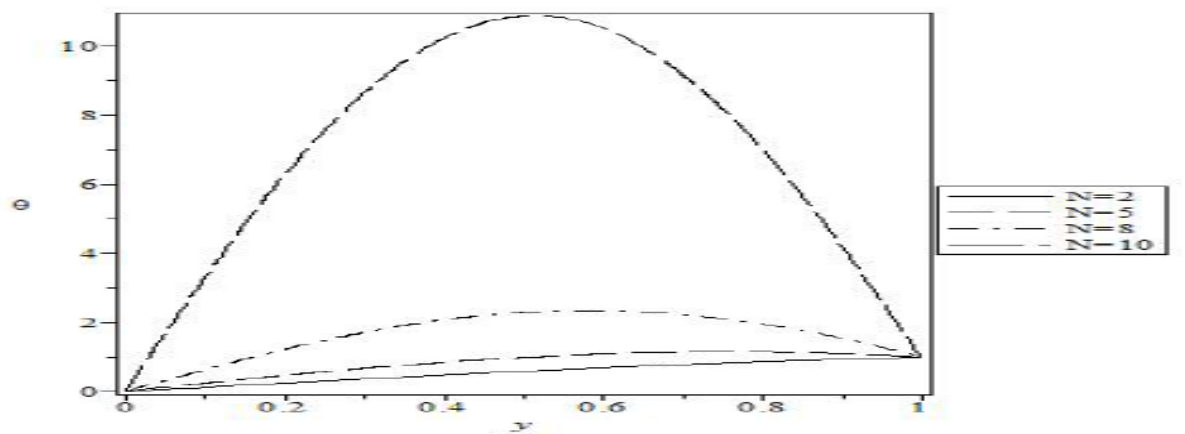

Figure 14: Temperature profile for different values of $N$

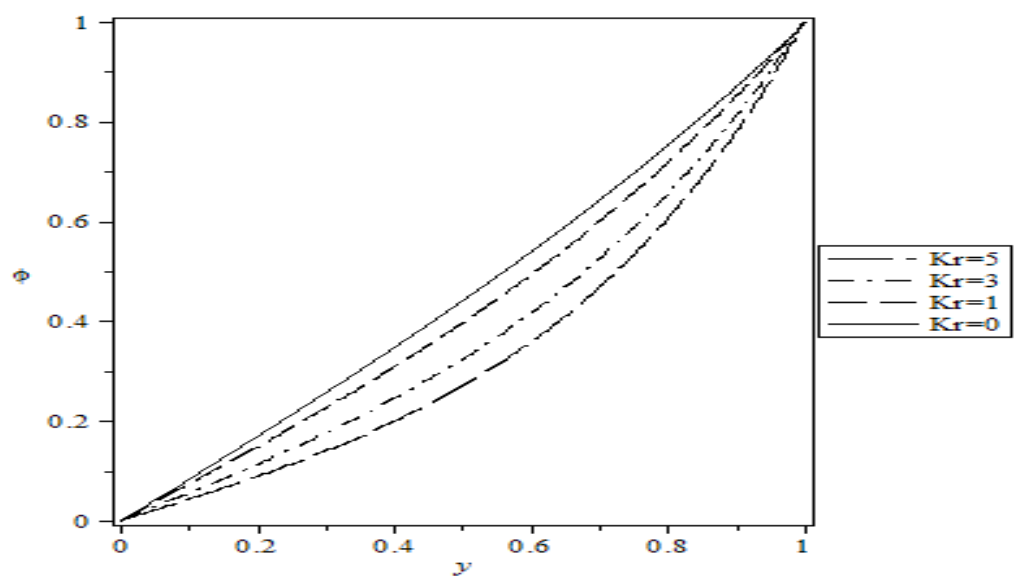

Figure 15: Concentration profile for different values of $K_{r}$

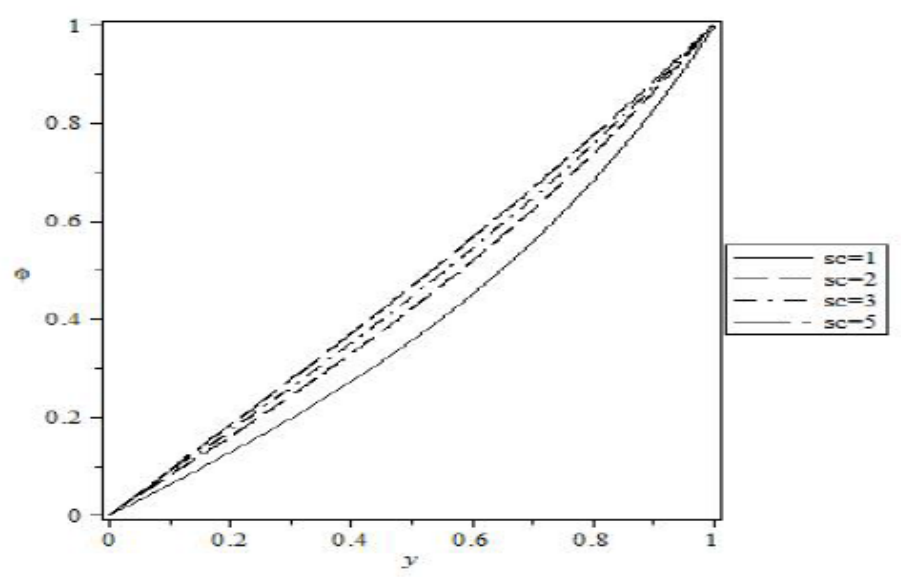

Figure 16: Concentration profile for different values of $S c$

Table 1: Effects of cold wall on the skin friction

\begin{tabular}{|l|l|l|l|l|l|l|l|}
\hline $\mathrm{y}$ & $\eta$ & $\gamma$ & $\tau$ & $\gamma$ & $\tau$ & $\gamma$ & $\tau$ \\
\hline 0 & 0.1 & 0 & -0.51681 & 0.2 & -0.36287 & 0.3 & -0.31631 \\
\hline 0.25 & 0.1 & 0 & -0.26822 & 0.2 & -0.17082 & 0.3 & -0.14101 \\
\hline 0.5 & 0.1 & 0 & 0.02321 & 0.2 & -0.08933 & 0.3 & 0.10957 \\
\hline 0.75 & 0.1 & 0 & 0.44234 & 0.2 & 0.49417 & 0.3 & 0.51001 \\
\hline 1 & 0.1 & 0 & 1.11678 & 0.2 & 1.6733 & 0.3 & 1.18281 \\
\hline
\end{tabular}


Table 2: Effects of heated wall on the skin friction

\begin{tabular}{|l|l|l|l|l|l|l|l|}
\hline $\mathrm{y}$ & $\eta$ & $\gamma$ & $\tau$ & $\eta$ & $\tau$ & $\eta$ & $\tau$ \\
\hline 0 & 0 & 0.1 & -0.47901 & 0.1 & -0.42666 & 0.2 & -0.33793 \\
\hline 0.25 & 0 & 0.1 & -0.27552 & 0.1 & -0.21102 & 0.2 & -0.10173 \\
\hline 0.5 & 0 & 0.1 & 0.03105 & 0.1 & 0.06204 & 0.2 & 0.21981 \\
\hline 0.75 & 0 & 0.1 & 0.32735 & 0.1 & 0.47281 & 0.2 & 0.71933 \\
\hline 1 & 0 & 0.1 & 0.91150 & 0.1 & 1.14647 & 0.2 & 1.54463 \\
\hline
\end{tabular}

Table 3: Effects of Peclet number on the skin friction

\begin{tabular}{|l|l|l|l|l|l|l|}
\hline $\mathrm{y}$ & $\mathrm{Pe}$ & $\mathrm{Nu}$ & $\mathrm{Pe}$ & $\mathrm{Nu}$ & $\mathrm{Pe}$ & $\mathrm{Nu}$ \\
\hline 0 & 0.044 & -1.41957 & 0.7 & -1.25476 & 1 & -1.18839 \\
\hline 0.25 & 0.044 & -1.33368 & 0.7 & -1.20409 & 1 & -1.15114 \\
\hline 0.5 & 0.044 & -1.08640 & 0.7 & -1.05629 & 1 & -1.04295 \\
\hline 0.75 & 0.044 & -0.70765 & 0.7 & -0.08233 & 1 & -1.86954 \\
\hline 1 & 0.044 & -0.24327 & 0.7 & 0.52368 & 1 & 0.64209 \\
\hline
\end{tabular}

Table 4: Effects ofScmidt number on Sherwood number

\begin{tabular}{|c|c|c|c|c|c|c|c|}
\hline$y$ & $K_{r}$ & $S C$ & $S h$ & $S C$ & $S h$ & $S c$ & $S h$ \\
\hline 0 & 1 & 1 & 0.73083 & 2 & 0.85092 & 3 & 0.89696 \\
\hline 0.25 & 1 & 1 & 0.77698 & 2 & 0.87765 & 3 & 0.91571 \\
\hline 0.5 & 1 & 1 & 0.92128 & 2 & 0.95951 & 3 & 0.97275 \\
\hline 0.75 & 1 & 1 & 1.18194 & 2 & 1.10167 & 3 & 1.07046 \\
\hline 1 & 1 & 1 & 1.59189 & 2 & 1.31304 & 3 & 1.10872 \\
\hline
\end{tabular}

Table 5: Effects of chemical reaction parameter on Sherwood number

\begin{tabular}{|l|l|l|l|l|l|l|l|}
\hline $\mathrm{y}$ & $S C$ & $K_{r}$ & $S h$ & $K_{r}$ & $S h$ & $K_{r}$ & $S h$ \\
\hline 0 & 1 & 0 & 0.85092 & 1 & 0.73042 & 2 & 0.63267 \\
\hline 0.25 & 1 & 0 & 0.87764 & 1 & 0.77693 & 2 & 0.69292 \\
\hline 0.5 & 1 & 0 & 0.95952 & 1 & 0.92128 & 2 & 0.88513 \\
\hline 0.75 & 1 & 0 & 1.10166 & 1 & 1.18194 & 2 & 1.24592 \\
\hline 1 & 1 & 0 & 1.31303 & 1 & 1.59189 & 2 & 1.84398 \\
\hline
\end{tabular}

\section{Conclusion}

This paper investigated the heat and mass transfers on MHD oscillatory slip flow in a channel with non-uniform wall temperature that is filled with porous medium. Exact solutions of the velocity,temperature and concentration are obtained. There is a good agreement between the present study and the effect of slip on MHD oscillatory presented by Mehmood and Ali [6] when $G c=\eta=0$ and the presented by Adesanya and Makinde when $\mathrm{Gc}=0$. However, Nusselt numberincreases at the cold plate but decreases at the heated plate with an increase in Peclet number.

\section{Refrences}

[1]. A.S. Eegunjobi and O.D. Makinde, Combined effect buoyancy force and Naiver slip on entropy generation in a Vertical porous channel, Entropy 14, 2012, 1028-1044.

[2]. O.D.Makinde and E. Osalusi, MHD steady flow in a channel with slip at the permeable boundaries, Rom. J.Physis 51 , 2006, 319 328.

[3]. A. Mehmood and A. Ali, The effect of slip condition on unsteady MHD oscillatory flow of a viscous fluid in a planner channel, Rom.J.Physis, 52, 2007,85-91.

[4]. S.O. Adesanya and O.D. Makinde, MHD oscillatory slip flow and Heat transfer in a channel filled with porous media, Rom.J.Physics, 76, 2014, 197-204.

[5]. D. Vijaya and G.R. Viswanadh, Effects of chemical reaction on MHD free convective oscillatory flow past a porous plate with dissipation and heat sink, Advance in Applied science Research, 3(5), 2012, 206-215.

[6]. N.B. Rabi,C.D. Gouranger and M. Artpita, Chemical reaction effects on MHD oscillatory slip flow through a porous medium bounded by two vertical porous plates with heat source and soret effect, Journal of Applied Analysis and computations, 3(4),2013, 234-243.

[7]. A.C.L. Cogley, W.G Vinvent and E.S. Giles, American Institute of Aeronautics and Astronautics, 1968, 551 